\title{
External fixation in patients with panvertebral osteomyelitis and paraplegia
}

\author{
ME Hurley ${ }^{1}$, AR Vaccaro ${ }^{2}$, GJ Scuderi ${ }^{1}$ and SR Garfin ${ }^{1}$ \\ ${ }^{1}$ University of California, San Diego, $200 \mathrm{~W}$ Arbor Drive 8894, San Diego, CA 92103 and ${ }^{2}$ Thomas Jefferson \\ University, 838 Walnut Street, Philadelphia, PA 19107, USA
}

\begin{abstract}
Vertebral osteomyelitis is a difficult problem in the spinal cord injured patient. We present three such cases treated with extensive debridement followed by grafting and stabilization with external fixation. In all three patients the infection resolved. We feel this is a viable option for treating this condition.
\end{abstract}

Keywords: osteomyelitis; panvertebral; paraplegia; external fixation; spinal cord injury

\section{Introduction}

Osteomyelitis is increasingly diagnosed in patients with spinal cord injury. Previously, it was a poorly recognized entity in this group of patients. ${ }^{1-5}$ The diagnosis of osteomyelitis is indeed difficult, as pain, which is the early symptom heralding its onset, is absent in patients with complete spinal cord injury. This fact, paired with a low index of suspicion entertained by many physicians who do not commonly treat these patients, contributes to the considerable delay in the diagnosis of vertebral osteomyelitis. In patients with prolonged delays, suppurative infections may develop with significant morbidity. ${ }^{6}$ Vertebral osteomyelitis with prevertebral and retroperitoneal abscesses in patients with paraplegia are relatively uncommon, but the occurrence is potentially catastrophic. These panvertebral infections are usually associated with decubitus ulcers and/or urinary tract infections. ${ }^{7}$ This report describes three patients who developed this condition and who were treated by extensive anterior and posterior debridement and external fixation of the spinal column.

\section{Methods}

Initial debridement of the spine is undertaken anteriorly through a retroperitoneal approach. A second contralateral retroperitoneal incision may be utilized because of the limited exposure offered by a unilateral approach. This is in part related to the extensive fibrosis and scarring of neural and vascular structures and the ureters along with calcification in the fibrotic iliopsoas muscles and abscess cavity which further limits mobilization. After debridement of the infection the spinal column architecture is assessed for grafting. Often significant cavities, secondary to the debridement are created, necessitating utilization of a

\footnotetext{
Correspondence: SR Garfin
}

large allograft strut. Wounds are then drained and closed and the patient is then turned prone under the same anesthetic. The spine is then approached posteriorly through a midline incision. Aggressive debridement is carried out and the pedicles are identified in the standard manner. AO $5.5 \mathrm{~mm}$ Shantz screws are placed in all appropriate pedicles. Poor bone stock in the sacrum may necessitate utilization of trans-ilial screws. These screws are placed in an oblique manner through the posterior superior iliac spine to engage both tables of the ilium. After fixation of all involved levels in the lumbar spine and either sacrum or ilium, the external framework is then assembled to form a rigid construction. The large forces transmitted to the lumbar spine necessitate the utilization of a 'trapezoidal' like construct. The posterior wound is packed open. Appropriate long-term parenteral, followed by oral, antibiotics are given, and the erythrocyte sedimentation rate (ESR) is followed.

\section{Case examples}

Case 1

A 33-year-old T12 paraplegic patient who had previously undergone posterior Harrington rod instrumentation first presented 13 years after his original injury with severe decubitus ulcers over the sacrum. Radiographs demonstrated retroperitoneal abscesses with L5 vertebral osteomyelitis. The patient underwent CT-guided percutaneous drainage, followed by a posterior debridement, removal of instrumentation, an L5 vertebrectomy, and fusion from L4 to the sacrum. Three years later he again presented with multiple decubiti, a urinary tract infection, and bilateral retroperitoneal abscesses with vertebral osteomyelitis and near complete destruction of the fourth and fifth lumbar vertebrae. The abscesses were drained through bilateral retroperitoneal approaches, 
and the patient underwent anterior L3 to the sacrum allograft strut arthrodesis. In order to stabilize the spine, the patient was then turned prone and external fixation was applied from L2 to the sacrum. The posterior wound was packed open. The patient was mobilized one week after surgery, and roentgenographic evaluation at 4 months showed early healing. $\mathrm{He}$ underwent removal of the external fixator, replacement with VSP plates and screw and refusion with allograft posteriorly. The wound was closed using a distally based gluteal musculo-cutaneous flap. At 4year follow-up the patient had a solid fusion of the lumbosacral spine with return to his previous level of function.

\section{Case 2}

A 35-year-old male patient had a 15-year history of $\mathrm{T} 7$ paraplegia with numerous episodes of decubiti and urinary tract infections. In June of 1988, the patient was diagnosed as having bilateral retroperitoneal

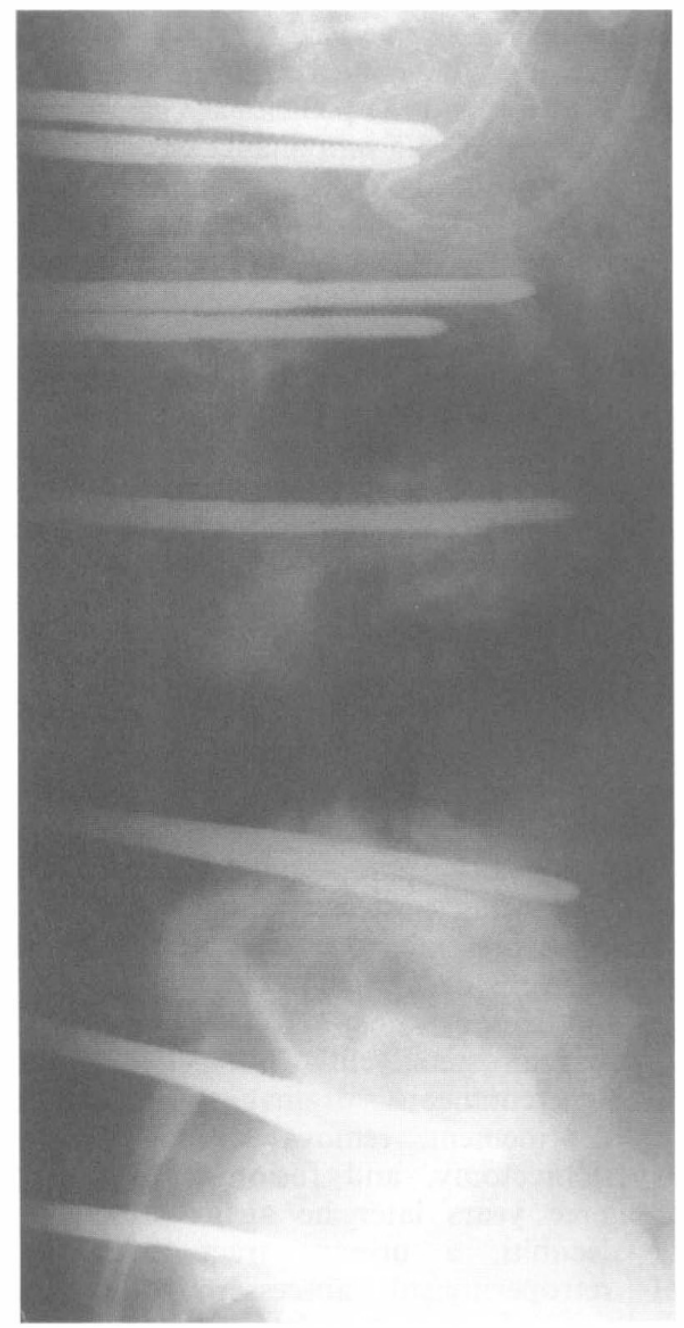

Figure 1 Case number 2 following debridement and placement of external fixation abscesses at L4 and L5 with concurrent osteomyelitis. The patient underwent drainage of the abscesses through bilateral retroperitoneal approaches with debridement and strut grafting. Posteriorly, the patient underwent debridement with application of an external fixator from $\mathrm{L} 1$ to the sacrum. The patient underwent early mobilization, and at 7 months postoperatively the external fixator was removed, Luque plates and pedicle screws were applied and the wound re-debrided and covered with a latissimus dorsi free flap. At 2-year follow-up, the patient has returned to his previous level of function with radiographic evidence of successful fusion (Figures 1, 2).

\section{Case 3}

A 30-year-old male with a 17-year history of T7 paraplegia developed a urinary tract infection subsequent to a diagnosis of vertebral osteomyelitis from L4 to the sacrum. Due to a significant delay in diagnosis, the patient developed bilateral retroperitoneal ab-

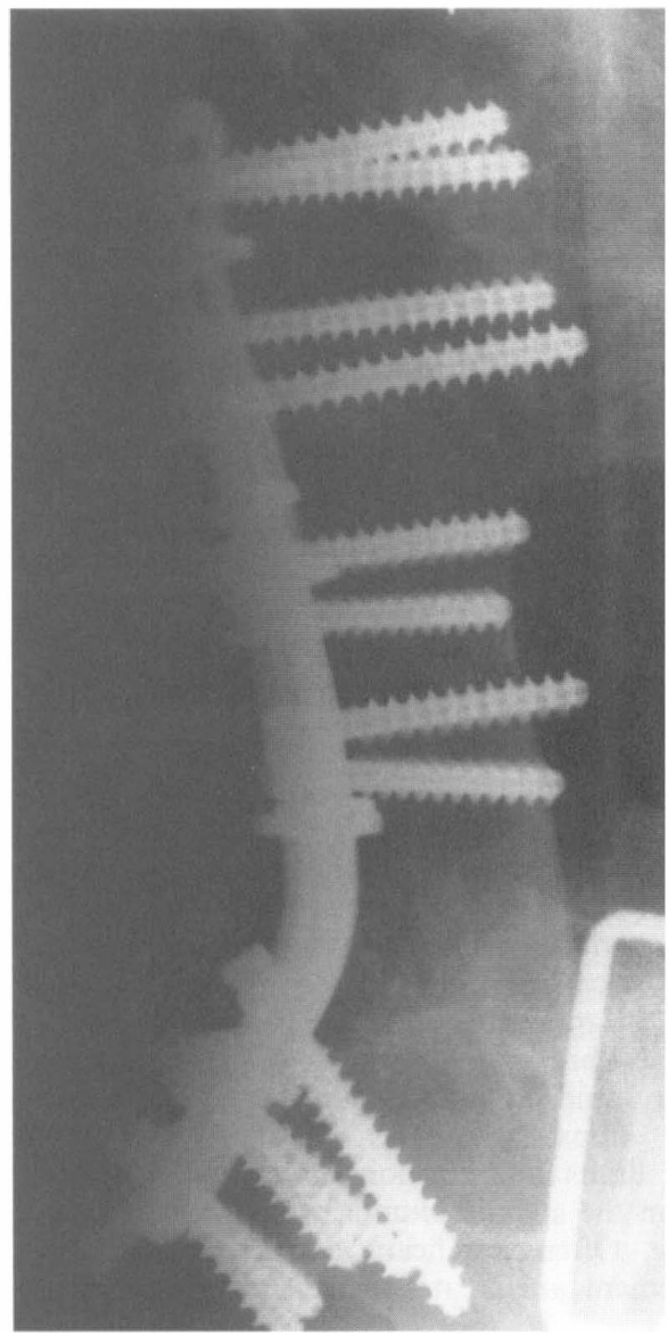

Figure 2 Case number 2 after incorporation of strut graft and internal fixation 
scesses. In November of 1987, these were drained through bilateral retroperitoneal approaches. The patient underwent L4 and L5 vertebrectomies with strut grafting. He was then turned prone and underwent fixation from L1 to the sacrum. Six months postoperatively, the patient had evidence of graft incorporation and the external fixation was removed and the wound closed with a distally based gluteal musculo-cutaneous flap. At 6-year follow-up, he has done well with no evidence of recurrence of his infection.

\section{Conclusion}

Osteomyelitis is an unfortunate occurrence in spinal cord injured patients. It is a serious disease, usually related to a contiguous focus (ie, a pressure sore). Diagnosis is difficult because many abnormal laboratory findings in patients with spinal cord injury are due to other conditions, and radiographic changes may be due to factors such as osteoporosis. ${ }^{8}$ Also, the lack of an appropriate pain response in this group of patients frequently causes considerable delay, increasing the morbidity. Numerous treatment options have been advocated in the care of patients with paraplegia who develop osteomyelitis. Intravenous antibiotics alone, or other combinations of surgical treatment, including posterior debridement and/or anterior debridement with or without grafting have been utilized with some success. ${ }^{9}$ However, previous treatment recommendations require prolonged hospitalization with significant periods of recumbency due to lack of spinal stability following treatment. The technique reported in this paper allows for rapid mobilization with early transfer to a rehabilitation facility at a significant decrease in cost, physical, mental, and financial.

Magerl was the first to report on external fixation in the treatment of vertebral osteomyelitis. He treated five patients with vertebral osteomyelitis with no complication. He stated that he did not apply external fixation in patients with paraplegia. He felt that the inability of the patient to cooperate was a contraindication for external fixation in this subgroup. ${ }^{10}$

In 1984, Magerl described his experience with external fixation of the spine in 65 patients, including eight with osteomyelitis. Analysis of the data in the eight patients with osteomyelitis showed good results in all. ${ }^{11}$

To our knowledge, no series has been reported on the treatment of patients with spinal cord injury and vertebral osteomyelitis. The three patients reported in this series all presented with recalcitrant infection, bilateral retroperitoneal abscesses, multi-level vertebral osteomyelitis, and early sepsis. All had failed previous surgical drainage and necessitated aggressive debridement and stabilization. All of the patients had a large amount of pus anteriorly, necessitating bilateral retroperitoneal incisions for debridement. After adequate debridement and drainage, strut grafting was performed anteriorly. Posterior stabilization was felt to be necessary due to the instability created by the bony destruction (infection and surgery).

Internal fixation in the presence of infection has not been considered a viable option in the acute setting. Internal fixation also decreases the space available for the application of bone graft. In this group of patients, external fixation proved to be an excellent treatment alternative in the stabilization of the vertebral column after massive debridement. It allowed wound care and early mobilization with the potential to decrease complications associated with prolonged recumbency. These cases illustrate the potential use of external fixation in the face of overwhelming spinal infection with the need for prolonged open wound care and stabilization of the spine.

\section{References}

1 Ross PM, Gleming JL. Vertebral Body Osteomyelitis. Clin Orthop 1976; 118: 190-198.

2 Griffiths HED, Jomes DM. Pyogenic Infection of the Spine. $J$ Bone Joint Surg 1971; 53B: 383-391.

3 Garcia A, Grantham SA. Hematogenous Pyogenic Vertebral Osteomyelitis. J Bone Joint Surg 1960; 42A: 429-436.

4 Frederickson B, Yuan H, Olans R. Management and Outcome of Pyogenic Vertebral Osteomyelitis. Clin Orthop 1978; 131: $160-$ 167.

5 Eismont FJ, Bohlman HH, Prasanna LS, Goldberg VM, Freehafer AA. Pyogenic and Fungal Vertebral Osteomyelitis with Paralysis. J Bone Joint Surg 1983; 65A: 19-29.

6 Wedge JH, Oryschak AF, Robertson DE, Kikaldy-Willis WH. Atypical Manifestations of Spinal Infection. Clin Orthop 1977; 123: $155-163$.

7 Henson SW, Coventry MB. Osteomyelitis of the Vertebrae as the Result of Infection of the Urinary Tract. Surgery, Gynecology, \& Obstetrics 1956; Feb: 207-214.

8 Eismont FJ, Bohlman HH, Prasanna LS, Goldberg VM, Freehafer AA. Pyogenic and Fungal Vertebral Osteomyelitis with Paralysis. J Bone Joint Surg 1983; 65A: 19-29.

9 Sanford EE, Donald PK, Chan, Woodward HR. Treatment of Hematogenous Pyogenic Vertebral Osteomyelitis with Anterior Debridement and Primary Bone Grafting. Spine 1989; 14: $284-$ 291.

10 Magerl F. External skeletal fixation of the lower thoracic and the lumbar spine. In Uhthoff, HK. (ed). Current Concepts of External Fixation of Fractures. Berlin, Heidelberg, SpringerVerlag, 1982; p. 353.

11 Magerl FP. Stabilization of the Lower Thoracic and Lumbar Spine with External Skeletal Fixation. Clin Orthp 1984; 189: 125. 